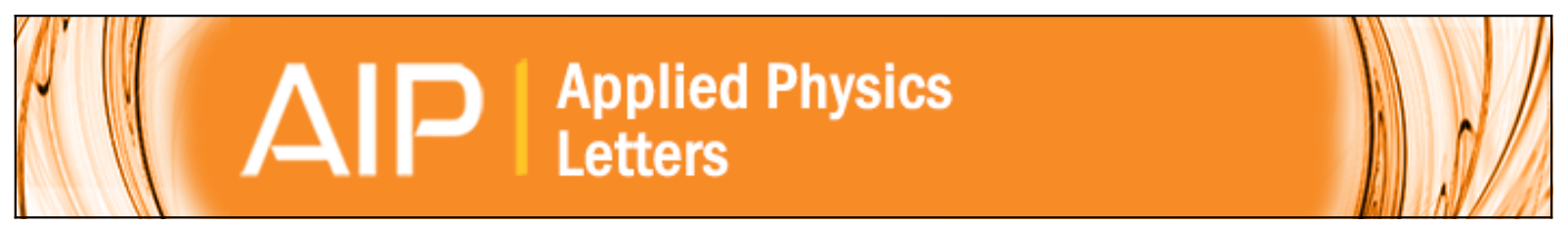

\title{
Thickness mapping of high- dielectrics at the nanoscale
}

J. Trapnauskas, M. Rommel, A. J. Bauer, and L. Frey

Citation: Applied Physics Letters 104, 052907 (2014); doi: 10.1063/1.4863947

View online: http://dx.doi.org/10.1063/1.4863947

View Table of Contents: http://scitation.aip.org/content/aip/journal/apl/104/5?ver=pdfcov

Published by the AIP Publishing

\section{Articles you may be interested in}

Coupling of Advanced Optical and Chemical Characterization Techniques for Optimization of High Dielectrics with Nanometer Range Thickness

AIP Conf. Proc. 931, 292 (2007); 10.1063/1.2799386

Nondestructive Thickness Determination of Highk Dielectric HfO2 and Interfacial Oxide by Spectroscopic Ellipsometry

AIP Conf. Proc. 788, 177 (2005); 10.1063/1.2062959

Highk dielectric characterization by VUV spectroscopic ellipsometry and Xray reflection AIP Conf. Proc. 683, 148 (2003); 10.1063/1.1622462

Advanced Characterization of High Materials Interfaces by HighResolution Photoemission using Synchrotron Radiation

AIP Conf. Proc. 683, 139 (2003); 10.1063/1.1622460

Germanium nanowire field-effect transistors with $\mathrm{SiO} 2$ and high- $\mathrm{HfO} 2$ gate dielectrics Appl. Phys. Lett. 83, 2432 (2003); 10.1063/1.1611644

\section{AlP Re-register for Table of Content Alerts}




\title{
Thickness mapping of high- $\kappa$ dielectrics at the nanoscale
}

\author{
J. Trapnauskas, ${ }^{1, a)}$ M. Rommel, ${ }^{1, b)}$ A. J. Bauer, ${ }^{1}$ and L. Frey ${ }^{1,2}$ \\ ${ }^{1}$ Fraunhofer Institute for Integrated Systems and Device Technology (IISB), Schottkystrasse 10, \\ 91058 Erlangen, Germany \\ ${ }^{2}$ Chair of Electron Devices, University of Erlangen-Nuremberg, Cauerstrasse 6, 91058 Erlangen, Germany
}

(Received 4 November 2013; accepted 19 January 2014; published online 4 February 2014)

\begin{abstract}
Contact potential difference measurements by Kelvin probe force microscopy (KPFM) together with corona charging was applied for contactless equivalent oxide thickness (EOT) mapping with nanometer range lateral resolution. Characterization was performed under ambient conditions and compared to a conventional macroscale method based on Kelvin probe measurements. The presented method is directly applicable for dielectrics with homogeneous thicknesses. For other dielectric layers, the implemented laboratory setup suffers from imprecise KPFM sample positioning. If such samples have microscopically non-flat surfaces a proposed data evaluation procedure overcomes that issue which is demonstrated for nanoscale EOT maps of $\mathrm{HfO}_{2}$ and HfSi $_{\mathrm{x}} \mathrm{O}_{\mathrm{y}}$ samples. C 2014 AIP Publishing LLC. [http://dx.doi.org/10.1063/1.4863947]
\end{abstract}

For process development or control of $\mathrm{SiO}_{2}$ based amorphous dielectrics, it is sufficient to record thickness maps at the macroscale (wafer scale) in a contactless manner with a lateral resolution of millimeters. Ellipsometry or interferometry is most widely used for this purpose. However, to get additional information about charges in the oxide or about interface state densities, methods are used which employ corona charging together with contact potential difference (CPD) measurements between the sample's surface and its backside. The idea behind such characterization is explained by Edelman et al. ${ }^{1}$ The equivalent oxide thickness (EOT) is evaluated by charging the sample at least twice with known densities of corona generated positive $\left(\mathrm{H}_{3} \mathrm{O}^{+}\right)$or negative $\left(\mathrm{CO}^{-3}\right)$ ions $^{2}$ and measuring the resulting change in $\mathrm{CPD}$ with a scanning Kelvin probe (SKP). If the sample is in accumulation throughout this procedure, EOT can be easily calculated from the corresponding differences in deposited charge density $(\Delta \mathrm{Q})$ and $\mathrm{CPD}(\Delta \mathrm{CPD})$ (see Eq. (1); $\mathrm{C}_{\mathrm{Is}}$ is the dielectric capacitance, $\varepsilon_{\mathrm{SiO} 2}$ and $\varepsilon_{0}$ are the relative permittivity of $\mathrm{SiO}_{2}$ and the vacuum permittivity, respectively) ${ }^{2}$

$$
E O T=\frac{\varepsilon_{\mathrm{SiO}_{2}} \varepsilon_{0}}{C_{I S}}=\frac{\varepsilon_{\mathrm{SiO}_{2}} \varepsilon_{0} \Delta C P D}{\Delta Q} .
$$

However, today, high- $\kappa$ insulators are widely used as gate dielectrics in semiconductor devices. In contrast to $\mathrm{SiO}_{2}$, depending on deposition temperature, post-deposition annealing condition, film thickness, etc., high- $\kappa$ layers are often nanocrystalline. Because of the formation of grains and grain boundaries which may lead to increased surface roughness, dielectric thickness fluctuations might occur, too. The lateral dimensions of such crystallites are rather small (typically some tens of nanometers). Even the best SKPs, however, reach only several microns as a maximum lateral resolution. ${ }^{3}$ Thus, a CPD measurement method with a

\footnotetext{
a) Present address: Institute for Microsystems Technology (IMTEK), Georges-Köhler-Allee 103, 79110 Freiburg, Germany.

b)Electronic mail: mathias.rommel@iisb.fraunhofer.de
}

significantly higher lateral resolution is required to detect thickness variations at the nanoscale.

Recently, using Kelvin probe force microscopy (KPFM), charge distribution within a single molecule has been imaged ${ }^{4}$ which proves the very high resolution of KPFM's surface potential measurements. It is an offspring of non-contact atomic force microscopy (AFM) where each scan line is scanned twice: In tapping mode to measure topography (height) and in lift mode to obtain CPD. During lift mode, an oscillating drive voltage is applied, not to the piezo as in tapping mode but to the tip itself whose DC voltage is adjusted by a feedback loop to nullify the tip-sample Coulomb force. ${ }^{5}$ In such case, the adjusted tip's DC voltage is equal to the CPD. Besides, Lägel et al. ${ }^{5}$ have already observed that only insignificant amount of charge is lost due to slightly tapping the sample's surface with an AFM tip. Also, they have compared CPD measurements performed with both, SKP in ambient and KPFM in $\mathrm{N}_{2}$ environment on differently corona charged $\mathrm{SiO}_{2}$ samples, and found a nearly perfect agreement, meaning that KPFM can be used in conjunction with a corona charging technique to properly detect CPD changes due to the corona charge. ${ }^{5}$ Therefore, KPFM instead of SKP should be applicable for the thickness mapping of high- $\kappa$ dielectrics with nanoscale lateral resolution which will be analyzed in this work.

As the used KPFM setup is operating in ambient rather than $\mathrm{N}_{2}$ environment, first, samples with laterally homogeneous $\mathrm{SiO}_{2}$ stripes are prepared to prove the agreement between macro- and nanoscale CPD measurements for the given setup, and, thus, that the given approach is capable to measure dielectric thicknesses. For this purpose, two $150 \mathrm{~mm}$-type $\mathrm{Si}$ wafers were thermally oxidized with an initial $\mathrm{SiO}_{2}$ thickness of $20 \mathrm{~nm}$. Subsequently, the samples (in the following referred to as $\mathrm{SiO}_{2} \mathrm{~A}$ and $\mathrm{SiO}_{2} \mathrm{~B}$, respectively) were partially etched to produce $\mathrm{SiO}_{2}$ stripes of different thickness (i.e., approximately $5,10,15$, and $20 \mathrm{~nm}$ as confirmed by ellipsometry). Second, three nanocrystalline high- $\kappa$ samples were prepared to investigate the lateral resolution of the method.

As the first high- $\kappa$ sample, a $10 \mathrm{~nm} \mathrm{HfO}_{2}$ layer was grown by 75 cycles of atomic layer deposition at $300^{\circ} \mathrm{C}$ on a 
$150 \mathrm{~mm}$-type Si wafer covered with $\sim 1 \mathrm{~nm}$ natural oxide layer. Subsequently, the wafer was rapid thermally annealed (RTA) in an $\mathrm{O}_{2}$ environment at $900{ }^{\circ} \mathrm{C}$ for $15 \mathrm{~s}$.

The other two high- $\kappa$ samples were prepared by growing a $4 \mathrm{~nm} \mathrm{SiO}{ }_{2}$ layer using dry thermal oxidation on a $150 \mathrm{~mm}$ $p$-type $\mathrm{Si}$ wafer. On top of it, a $5 \mathrm{~nm} \mathrm{HfSi}_{\mathrm{x}} \mathrm{O}_{\mathrm{y}}$ layer with slightly higher thickness towards the edge of the wafer was deposited by metal organic chemical vapor deposition. The wafer was then annealed by RTA at $900^{\circ} \mathrm{C}$ in $\mathrm{O}_{2}$ for $10 \mathrm{~s}$. Finally, the wafer was broken into halves, and one half was further annealed at $450^{\circ} \mathrm{C}$ in forming gas for $30 \mathrm{~min}$. The latter will be referred to as "RTA + FGA" and the former as "RTA." For sample "RTA," measurements were performed close to the center of the wafer whereas for sample "RTA + FGA," a measurement position closer to the wafer edge was chosen (i.e., higher thickness compared to sample "RTA").

The procedure for comparing macro- and nanoscale CPD measurements, as well as estimated thicknesses, is summarized as follows: First, the sample was brought into deep accumulation by uniformly charging its surface with a linear corona charger of a macroscale tool (Semilab WT-2500). Then, the CPD values at the spots of interest (and, if relevant, their change with time) were measured with the same tool using SKP ( $8 \mathrm{~mm}$ diameter Kelvin probe). Immediately after, the sample was transferred to the AFM laboratory and within the spot area of the macroscopic tool, CPD was measured at the nanoscale with KPFM using a lift height of $10 \mathrm{~nm}$ and a tip drive amplitude of $1 \mathrm{~V}$ (Bruker Dimension Icon in ambient conditions, Nanoscope $\mathrm{V}$ controller, PtIr coated EFM probes for $\mathrm{SiO}_{2}$ and SSS-NCH probes for high- $\kappa$ samples, both NANOSENSORS ${ }^{\mathrm{TM}}$ ). Then, the sample was transferred back to the macroscale tool, and CPD was measured again to detect any charge leakage during KPFM and sample transfer, in which case leakage could be corrected for in the thickness determination procedure presented later. The sample was charged again with a known charge density, and the



FIG. 1. Comparison of macro- and nanoscale CPD measurements for samples $\mathrm{SiO}_{2} \mathrm{~A}$ and $\mathrm{SiO}_{2} \mathrm{~B}$ with stripes of different thicknesses $t_{\mathrm{Ox}}$ for different amounts of corona deposited charge (symbols). Lines correspond to linear regressions for each data set (regression coefficients of 1.0 each).
TABLE I. Comparison of average SKP and KPFM EOTs for the $\mathrm{SiO}_{2}$ samples together with the difference of the KPFM EOTs with respect to the SKP EOTs.

\begin{tabular}{lcccc}
\hline \hline & \multicolumn{3}{c}{ Thickness (nm) } & \\
\cline { 2 - 4 } Sample & Stripe & SKP & KPFM & Difference (\%) \\
\hline $\mathrm{SiO}_{2} \mathrm{~A}$ & 5 & 5.3 & 5.8 & 9.4 \\
& 10 & 10.0 & 10.7 & 7.0 \\
& 15 & 14.9 & 15.6 & 4.7 \\
& 20 & 20.9 & 22.0 & 5.3 \\
$\mathrm{SiO}_{2} \mathrm{~B}$ & 5 & 5.1 & 5.1 & 0 \\
& 10 & 10.8 & 10.4 & 3.7 \\
& 15 & 15.8 & 14.9 & 5.7 \\
& 20 & 20.8 & 20.1 & 3.4 \\
\hline \hline
\end{tabular}

above described measurements were performed (i.e., SKP and KPFM CPD measurements). This cycle was repeated 2-3 times and EOT was obtained from the slope of the linear fit of the dependence of CPD on corona charge. ${ }^{1}$

The comparison of SKP and KPFM CPD measurements for the $\mathrm{SiO}_{2}$ samples is provided in Fig. 1. A very good correlation between macro- and nanoscale CPD values can be observed for both samples and for all thicknesses. Note that CPD is a relative not absolute quantity and the regression offsets for both samples are not equal because the Kelvin probe of the macroscopic tool and the KPFM tips used for each sample had different surface potentials.

The average EOTs for the $\mathrm{SiO}_{2}$ samples as evaluated from the described measurements are given in Table I. It can be seen that the absolute relative differences are mostly low (3\%-6\%), with a maximum being $9.4 \%$ for the $5 \mathrm{~nm}$ stripe of sample $\mathrm{SiO}_{2} \mathrm{~A}$. The differences are caused by the fluctuating surface potential of the KPFM tip ${ }^{5}$ and charge leakage.

Next, it was aimed at determining thickness maps with nanoscale lateral resolution for high- $\kappa$ samples. From the corresponding KPFM measurements, a strong correlation
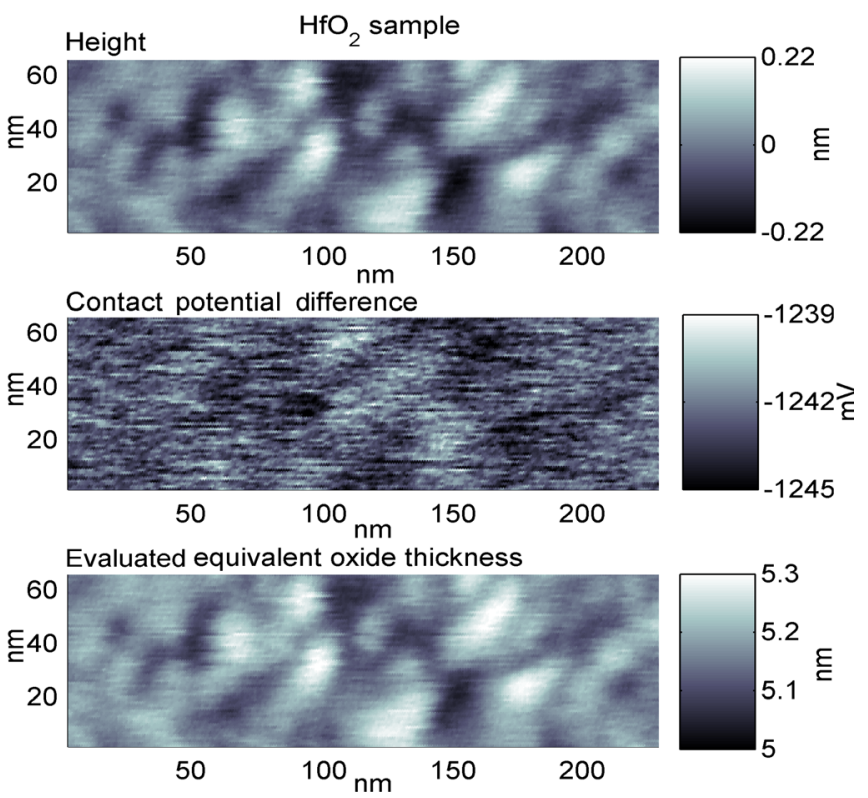

FIG. 2. Height and CPD map of sample $\mathrm{HfO}_{2}$ after first positive charging with $83.3 \mathrm{nC} / \mathrm{cm}^{2}$ together with the corresponding evaluated EOT map. Please note the different scale for height and EOT, respectively. 
between topography and CPD was observed: Smaller heights correspond to less negative CPD values and larger heights correspond to more negative CPD values (see Figs. 2 and 3). Similar results have been detected by Ludeke and Gusev ${ }^{6}$ who did not explain their findings. However, for the $\mathrm{SiO}_{2}$ samples, the likewise observed correlation between height and CPD is obviously due to a direct correlation between measured height and thickness. This should also hold for the high- $\kappa$ samples. Such hypothesis might be supported by the observation that in contrast to accumulation conditions, which were used so far, no correlation between height and CPD could be observed for depletion conditions. There, CPD is mainly governed by the contribution of space charges and trapped charges in the dielectric or at the interface and not by the dielectric capacitance (and, thus, dielectric thickness).
It should be noted that if corona charging could be performed at the KPFM tool in-situ without moving the sample, samples with laterally inhomogeneous thicknesses could be simply characterized as described for the $\mathrm{SiO}_{2}$ samples. Due to the required realignment of the sample during the described procedure, however, it is practically impossible to measure exactly the same nanoscopic area with the KPFM. To overcome this problem, the corona-Kelvin method was adapted to nanoscale. The adaptation assumes linearity between height and CPD and that the layer properties in general do not change laterally substantially within micrometers (experimentally determined AFM positioning accuracy is $\pm 1 \mu \mathrm{m})$. A scheme of the adapted method is provided in Fig. 3 with actual data for sample $\mathrm{HfSi}_{\mathrm{x}} \mathrm{O}_{\mathrm{y}}$ "RTA + FGA" and is explained in the following.

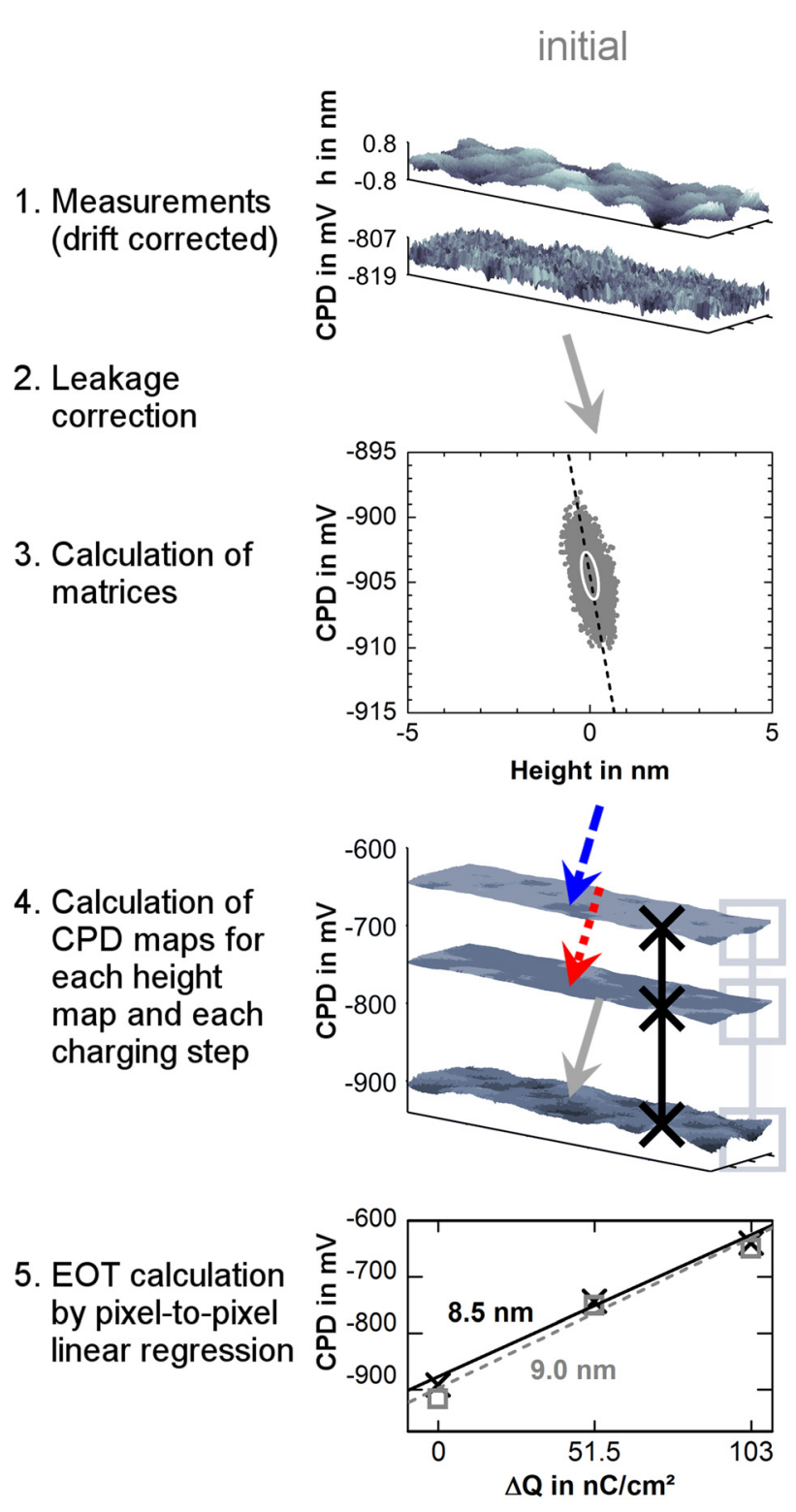

\section{after charging $51.5 \mathrm{nC} / \mathrm{cm}^{2}$}
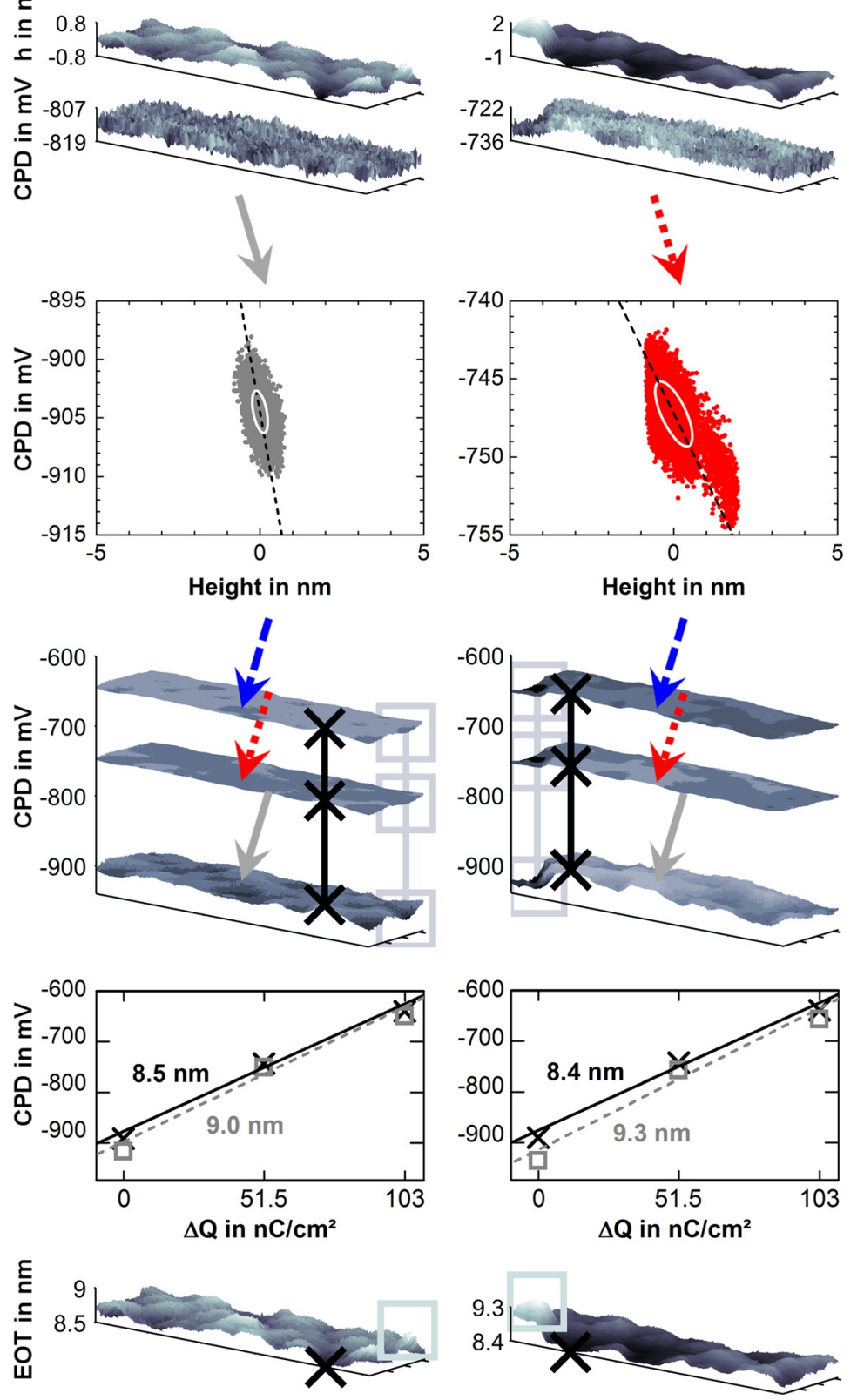

\section{after charging $51.5 \mathrm{nC} / \mathrm{cm}^{2}$ more}
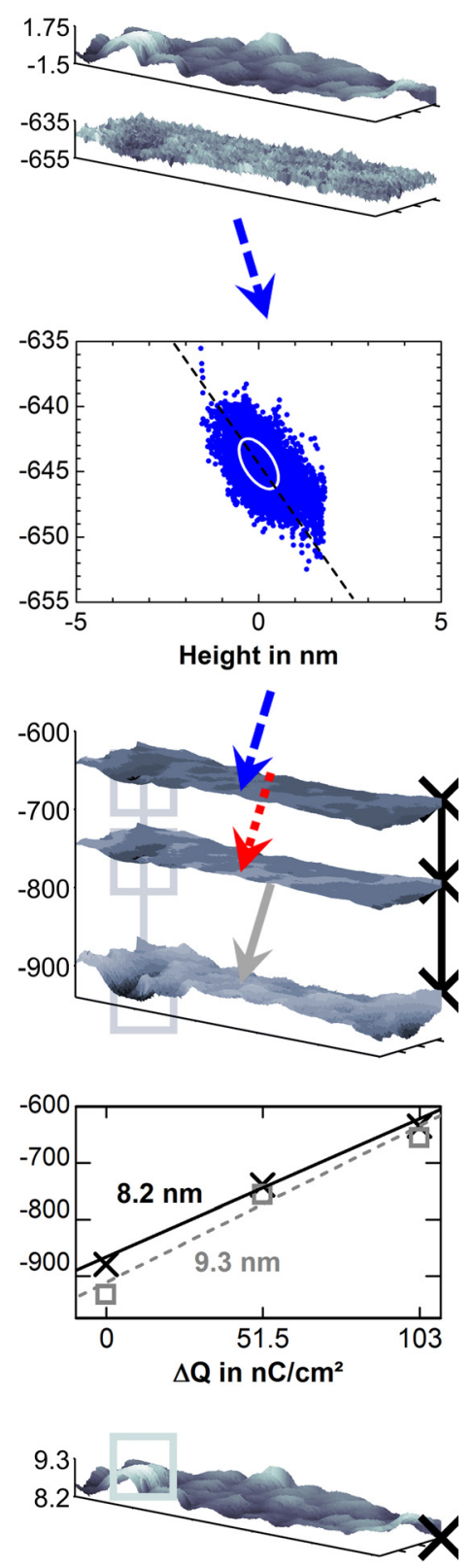

FIG. 3. Scheme of the adapted method using measured data for sample $\mathrm{HfSi}_{\mathrm{x}} \mathrm{O}_{\mathrm{y}}$ "RTA + FGA." 
KPFM measurements are performed at the same microscopic (but slightly different nanoscopic) spot after each corona charging $\Delta Q$ (step 1 in Fig. 3). Because height $h$ and CPD are measured by separately scanning the same line, a small drift might occur between topography and CPD map. The measured image pairs are corrected for it so that height peaks coincide precisely with CPD troughs and vice versa. Also, the CPD images are corrected for leakage by an amount which was determined by measuring the decay of CPD for approximately $90 \mathrm{~s}$ right after charging with the macroscopic tool and extrapolating the change in CPD due to leakage for the KPFM measurement date (step 2). Then, CPD vs. height relation is extracted for each measurement. Simple linear regression cannot be used here, because least squares method does not appropriately filter out any outliers and, therefore, provides an incorrect slope. Instead, CPD vs. height covariance matrices are calculated for each deposited charge density and the longer axes of the corresponding ellipses (first principal components) ${ }^{8}$ are regarded as filtered CPD vs. height regression slopes (step 3). These slopes were extracted for the height and CPD image pairs for each charge density are used to recalculate the CPD maps, corresponding to each of the surface charge depositions for every measured height map (in the example, for each height map three CPD maps are recalculated, step 4). Step 3 and step 4 are explained in more detail in Ref. 9. Finally, EOT for each pixel is calculated from a linear regression of the CPDs at that pixel vs. deposited charge density (step 5, exemplarily given for the points with smallest and largest thickness) to evaluate the EOT maps for sample $\mathrm{HfSi}_{\mathrm{x}} \mathrm{O}_{\mathrm{y}}$ "RTA + FGA" for each measurement (step 6).

According to the presented scheme, EOT maps for the other high- $\kappa$ samples could be also evaluated. Exemplarily, Fig. 2 shows results for sample $\mathrm{HfO}_{2}$ after charging the sample with $88.3 \mathrm{nC} / \mathrm{cm}^{2}$ (initial accumulation conditions were obtained for charging with $-596 \mathrm{nC} / \mathrm{cm}^{2}$ ). The noise visible in the CPD image is due to measurements being performed in ambient conditions. By taking the first principal component ${ }^{8}$ as the slope for recalculating the CPD images, the influence of such noise is minimized. Table II summarizes the evaluated average EOTs for the high- $\kappa$ samples which show a very good correlation between the results from both methods. The high average EOT of sample $\mathrm{HfSi}_{\mathrm{x}} \mathrm{O}_{\mathrm{y}}$ "RTA + FGA" compared to sample $\mathrm{HfSi}_{\mathrm{x}} \mathrm{O}_{\mathrm{y}}$ "RTA" results from the inhomogeneous thickness of the grown $\mathrm{HfSi}_{\mathrm{x}} \mathrm{O}_{\mathrm{y}}$ layer.

Obviously, the presented adapted method has limitations. The first one arises from the assumption of a linear relation between CPD and height. The thickness variations must have

TABLE II. Comparison of average SKP and KPFM EOTs together with the difference of the KPFM EOTs with respect to the SKP EOTs.

\begin{tabular}{lccc}
\hline \hline & \multicolumn{2}{c}{ Thickness (nm) } & \\
\cline { 2 - 3 } Sample & SKP & KPFM & Difference (\%) \\
\hline $\mathrm{HfO}_{2}$ & 5.3 & 5.2 & 1.9 \\
$\mathrm{HfSi}_{\mathrm{x}} \mathrm{O}_{\mathrm{y}}$ RTA & 5.7 & 5.7 & 0 \\
$\mathrm{HfSi}_{\mathrm{x}} \mathrm{O}_{\mathrm{y}}$ RTA + FGA & 8.5 & 8.7 & 2.4 \\
\hline \hline
\end{tabular}

a noticeable signature in topography (see, e.g., topography maps Figs. 2 and 3) which is typical for most high- $\kappa$ layers: Samples with smooth dielectric layer/substrate interface and rough surface, as well as those with both of them being rough could be properly evaluated. On the other hand, thickness variations would be impossible to evaluate for samples with flat surfaces. The majority of identical height points and noisy CPD data (low signal fluctuation to noise ratio) would cause very steep filtered $\mathrm{CPD} /$ height regression slopes and consequentially result in an incorrect evaluated thickness.

The second limitation comes from using the corona charging, as the ions deposited are actually contaminants. At the nanoscale, this is of high importance because the ions are of comparable size to the features and care must be taken when choosing the charge density. If it is too large, the ions cover the valleys of surface and, therefore, topography as well as CPD features can no longer be observed. Directly charging the surface with an AFM tip could possibly overcome this issue and has been already performed and thoroughly studied by Mesquida. ${ }^{7}$ Very low charge density, on the other hand, causes only a negligible change in filtered $\mathrm{CPD} /$ height regression slope which would bring the recalculated CPD maps too close together on a CPD scale and would result in significantly high thickness errors. These regression slopes should decrease monotonically when negatively charging and vice versa. This can be easily monitored after a new measurement has been performed and, in case of a slope change being, e.g., too small, the sample could be further charged. Also, having measured image pairs for more charging steps or having measured more times for the same deposited charge density would improve the final results.

Summarizing, average EOT values for $\mathrm{SiO}_{2}$ and high- $\kappa$ samples were evaluated in a contactless manner using corona-Kelvin metrology with SKP and KPFM applying a proposed evaluation procedure. EOT values from both methods were found to be in close agreement. Furthermore, thickness maps with nanoscale lateral resolution of annealed $\mathrm{HfO}_{2}$ and $\mathrm{HfSi}_{\mathrm{x}} \mathrm{O}_{\mathrm{y}}$ samples could be determined in ambient conditions without a need for a very precise sample positioning and were exemplarily presented. Thus, it is proven that a macroscale thickness mapping method was adapted to nanoscale. Finally, method's limitations were discussed and possible workarounds provided.

The authors gratefully acknowledge the support of NanoWorld Services GmbH for providing the probes used within this work.

${ }^{1}$ P. Edelman, A. Savtchouk, M. Wilson, J. D'Amico, J. N. Kochey, D. Marinskiy, and J. Lagowski, Eur. Phys. J.: Appl. Phys. 27, 495 (2004).

${ }^{2}$ D. K. Schroder, Meas. Sci. Technol. 12, R16 (2001).

${ }^{3}$ L. Kronik and Y. Shapira, Surf. Sci. Rep. 37, 1 (1999).

${ }^{4}$ F. Mohn, L. Gross, N. Moll, and G. Meyer, Nat. Nanotechnol. 7, 227 (2012).

${ }^{5}$ B. Lägel, M. D. Ayala, and R. Schlaf, Appl. Phys. Lett. 85, 4801 (2004).

${ }^{6}$ R. Ludeke and E. Gusev, J. Appl. Phys. 96, 2365 (2004).

${ }^{7}$ P. Mesquida, Ph.D. thesis, ETH Zürich, Zürich, 2002.

${ }^{8}$ I. T. Jolliffe, Principal Component Analysis, Springer Series in Statistics, 2nd ed. (Springer, 2002).

${ }^{9}$ See supplementary material at http://dx.doi.org/10.1063/1.4863947 for details of the proposed evaluation procedure including exemplary numerical data. 